\title{
Serodiagnosis of human neurocysticercosis using antigenic components of Taenia solium metacestodes derived from the unbound fraction from jacalin affinity chromatography
}

\author{
Gleyce Alves Machado ${ }^{1,2}$, Heliana Batista de Oliveira ${ }^{1,2}$, \\ Margareth Leitão Gennari-Cardoso ${ }^{1,3}$, José Roberto Mineo 1 , Julia Maria Costa-Cruz ${ }^{1+}$ \\ 1Departamento de Imunologia, Microbiologia e Parasitologia, Instituto de Ciências Biomédicas, Universidade Federal de Uberlândia, \\ Uberlândia, MG, Brasil ²Departamento de Ciências Biológicas, Universidade Federal de Goiás, Campus Catalão, Catalão, \\ GO, Brasil ${ }^{3}$ Departamento de Ciências Biológicas, Universidade Estadual de Santa Cruz, Ilhéus, BA, Brasil
}

The aim of the present study was to analyse Taenia solium metacestode antigens that were derived from the unbound fraction of jacalin affinity chromatography and subsequent tert-octylphenoxy poly(oxyethylene)ethanol Triton X-114 (TX-114) partitioning in the diagnosis of human neurocysticercosis (NCC). Immunoassays were designed to detect T. solium-specific IgG antibodies by ELISA and immunoblot. Serum samples were collected from 132 individuals who were categorised as follows: 40 had NCC, 62 presented Taenia spp or other parasitic diseases and 30 were healthy individuals. The jacalin-unbound $\left(J_{\text {unbound }}\right)$ fraction presented higher sensitivity and specificity rates than the jacalin-bound fraction and only this fraction was subjected to subsequent TX-114 partitioning, resulting in detergent $\left(D J_{\text {unbound }}\right)$ and aqueous $\left(A J_{\text {unbound }}\right)$ fractions. The ELISA sensitivity and specificity were $85 \%$ and $84.8 \%$ for $J_{\text {unbound }} 92.5 \%$ and $93.5 \%$ for $D J_{\text {unbound }}$ and $82.5 \%$ and $82.6 \%$ for $A J_{\text {unbound }}$. By immunoblot, the DJ unbound fraction showed $100 \%$ sensitivity and specificity and only serum samples from patients with NCC recognised the $50-70 \mathrm{kDa}$ T. solium-specific components. We conclude that the DJ unbound fraction can serve as a useful tool for the differential immunodiagnosis of NCC by immunoblot.

Key words: neurocysticercosis - Taenia solium - jacalin - Triton X-114 - diagnosis

Neurocysticercosis (NCC) is caused by infection with Taenia solium metacestodes and is an important cause of neurological diseases worldwide. NCC is commonly associated with seizures, headaches and focal neurological deficits and can have such sequelae as epilepsy, hydrocephalus and dementia (Agapejev 2011, Carabin et al. 2011). This disease is distributed globally, particularly in Central and South America, India, Sub-Saharan Africa, East Asia, Eastern Europe and other developing countries (Garcia et al. 2010, Afonso et al. 2011, Prasad et al. 2011, Yanagida et al. 2012).

The diagnosis of NCC on clinical grounds is difficult because its signs and symptoms are non-specific. An accurate diagnosis requires a combination of clinical, epidemiologic, imaging and immunologic information (Nash \& Garcia 2011). Imaging information, although very sensitive and specific, is not always available. For these reasons, immunoassays that are standardised for a proper diagnosis of NCC will lead to better clinical management of NCC patients because the immunoassays support diagnoses for patients whose clinical and imaging profiles are compatible with NCC (Michelet et

Financial support: CAPES, CNPq, FAPEMIG

+ Corresponding author: costacruz@ufu.br

Received 26 September 2012

Accepted 23 November 2012 al. 2011). Among various immunological methods, the ELISA and immunoblot are the most frequently used to detect antibodies against $T$. solium antigens in serum or cerebrospinal fluid samples, but these tests have shown different degrees of sensitivity and specificity, depending on the method of antigen preparation used (Shiguekawa et al. 2000, Barcelos et al. 2007, Deckers \& Dorny 2010, Gonçalves et al. 2010, Lee et al. 2011, Michelet et al. 2011, Ferrer et al. 2012).

Jacalin, a major protein from the seeds of the jackfruit Artocarpus integrifolia, is a tetrameric two-chain lectin with a molecular mass of 60-66 kDa. This glycoprotein binds only to O-glycosidically linked oligosaccharides and prefers to bind galactosyl $(\beta-1,3) \mathrm{N}$-acetylgalactosamine.

Lectins are proteins that possess at least one noncatalytic domain, which binds reversibly to a specific monosaccharide through hydrogen bonds, metal coordination, van der Waals interactions and hydrophobic interactions (Kabir 1998, Wu et al. 2003). This sugarbinding property of lectins also confers the capacity to be isolated by affinity chromatography on matrices containing their specific sugar ligand. Affinity separation techniques are based on biospecific molecular interactions. Therefore, they are extremely powerful tools for the isolation of valuable biological macromolecules (Jeyaprakash et al. 2005).

A solution of the nonionic surfactant Triton X-114 (TX-114) is homogeneous at $0^{\circ} \mathrm{C}$ but separates into detergent (D) and aqueous (A) phases above $20^{\circ} \mathrm{C}$. The efficiency of this separation increases with temperature 
and is sensitive to the presence of other surfactants. Integral membrane proteins of an amphiphilic nature are recovered in the $\mathrm{D}$ phase and hydrophilic proteins are found exclusively in the A phase (Bordier 1981). T. solium metacestode proteins recovered in the D phase have shown good results by ELISA and immunoblot for the diagnosis of NCC, but antigens purified using this technique alone have also shown some cross-reactivity, especially with Echinococcus granulosus by ELISA (Machado et al. 2007).

The major aim of this study was to isolate the antigenic components from a saline extract of $T$. solium metacestodes by jacalin affinity chromatography followed by TX114 partitioning and to evaluate the antigenicity degree of these fractions in the detection of $\mathrm{IgG}$ antibodies by ELISA and immunoblot for the diagnosis of human NCC. After sodium dodecyl sulfate polyacrylamide gel electrophoresis (SDS-PAGE) analysis, our findings demonstrated that the jacalin-unbound ( $\left.\mathrm{J}_{\text {unbound }}\right)$ fraction showed to be more specific than the jacalin-bound ones and therefore, only this fraction was subjected to TX-114 partitioning.

\section{SUBJECTS, MATERIALS AND METHODS}

Serum samples - Serum samples were collected from 132 subjects who were selected by the Laboratory of Clinical Analysis of the Clinical Hospital (Groups 1 and 2) and the Laboratory of Parasitology (Group 3) of the Federal University of Uberlândia in the state of Minas Gerais (MG), Brazil. Group 1 was composed of 40 patients who had been diagnosed with definitive NCC based on the presence of clinical symptoms, epidemiological data, positive immunological tests and evidence of the parasite by computerised tomography, as follows: (i) clinical syndrome: all patients presented at least one type of clinical manifestation that was suggestive of NCC, including epilepsy $(55 \%)$, cephalea $(50 \%)$, dizziness $(27.5 \%)$, dementia $(12.5 \%)$, faintness $(10 \%)$ and hydrocephalus $(2.5 \%)$ and no signs or symptoms that were suggestive of the presence of metacestodes in other organs were present; (ii) epidemiological data: all patients came from or lived in an area where cysticercosis is endemic, as previously described (Barcelos et al. 2012), in addition to at least two instances of household contact with T. solium infection; (iii) immunological diagnosis: cerebrospinal fluid samples were positive for anti-cysticercal IgG antibodies by ELISA; (iv) cerebral computerised tomographic findings: all patients presented evidence of the parasite by neuroimaging with the following classifications based on Sotelo et al. 1985: eight (20\%) vesicular, $15(37.5 \%)$ vesicular/ calcified and 17 (42.5\%) calcified metacestodes. According to the Del Brutto diagnostic criteria, all patients from Group 1 had a definitive diagnosis; 29 (72.5\%) had the absolute criteria and $11(27.5 \%)$ presented two major plus one minor or epidemiologic criteria (Del Brutto 2012). Of the patients who presented with the active form of NCC $(n=23), 16(70 \%)$ had the absolute criteria, whereas of those who presented with calcified lesions $(\mathrm{n}=17), 13$ (76\%) had the absolute criteria.

Group 2 was composed of 62 patients who were inflicted with Taenia spp adult intestinal worms (10) and other parasites (52), according to the following distribu- tion: Ascaris lumbricoides (6), E. granulosus (10), Enterobius vermicularis (6), Giardia lamblia (4), hookworm (6), Hymenolepis nana (4), Schistosoma mansoni (10), Strongyloides stercoralis (4) and Trichuris trichiura (2). With the exception of the E. granulosus-infected patients, all members of Group 2 were diagnosed by the ether sedimentation technique for routine stool examinations, as described by Ritchie (1948). The E. granulosus-infected patients were diagnosed by clinical assessment as well as by imaging and histopathological techniques. Group 3 was composed of 30 healthy volunteers, based on their clinical records. Although they came from areas that are endemic for cysticercosis, none of them presented any evidence of household contact with $T$. solium infection or a previous history of taeniasis or cysticercosis. In addition, three faecal samples from these individuals tested negative by the parasitological methods of Lutz (1919) and Baermann (1917).

Ethics - This study was approved by the Research Ethical Committee of the Federal University of Uberlândia, MG.

Parasites - Fresh T. solium metacestodes were collected from infected pig muscle and carefully dissected from the host tissues, washed repeatedly and stored at $-20^{\circ} \mathrm{C}$. The saline extract was prepared as described by Costa et al. (1982), with modifications. Briefly, 50 metacestodes were disrupted in $5 \mathrm{~mL}$ distilled water in an ice bath for $5 \mathrm{~min}$, homogenised for $4 \mathrm{~min}$ and subsequently submitted to ultrasonic treatment (Thornton, Inpec Electronics, Valinhos, São Paulo, Brazil) at $40 \mathrm{kHz}$ for four cycles of $30 \mathrm{~s}$ each in an ice bath. After isotonisation in $5 \mathrm{~mL}$ of $0.3 \mathrm{M} \mathrm{NaCl}$, the mixture was again submitted to ultrasonic treatment as described above and then stirred at $4^{\circ} \mathrm{C}$ for $2 \mathrm{~h}$ and centrifuged at $12,400 \mathrm{~g}$ and $4^{\circ} \mathrm{C}$ for 30 min (Du Pont SORVALL Products, Newtown, Connecticut, USA). The supernatants were analysed for protein content as described by Lowry et al. (1951) and stored in aliquots at $-70^{\circ} \mathrm{C}$ until use.

Isolation of jacalin-reactive components from saline extracts of T. solium metacestodes - The jacalin-reactive components were isolated according to Hermanson et al. (1995) with some modifications. First, the glycosylated proteins were isolated from saline extracts of $T$. solium metacestodes (12 mg total protein) by affinity chromatography using $5 \mathrm{~mL}$ jacalin immobilised on cross-linked 4\% agarose (Sigma-Aldrich Chemical Co, St. Louis, Missouri, USA) previously equilibrated with phosphate-buffered saline (PBS). The column was locked and incubated overnight under continuous slow agitation at $4^{\circ} \mathrm{C}$. The unbound material was washed with PBS and the jacalinbound components were eluted with $0.4 \mathrm{M}$ D-galactose (Sigma) at a flow rate of $0.5 \mathrm{~mL} / \mathrm{min}$, monitored by absorbance at $280 \mathrm{~nm}$ and collected in $2 \mathrm{~mL}$ fractions. The jacalin-bound and $J_{\text {unbound }}$ fractions were independently concentrated using a stirred ultrafiltration cell (Amicon, YM-10, WR Grace \& Co) and the jacalin-bound fraction was dialysed against PBS. The protein content of each antigen preparation was determined according to Lowry et al. (1951) and corresponded to $0.5 \mathrm{mg} / \mathrm{mL}$ for jacalinbound and $3 \mathrm{mg} / \mathrm{mL}$ for $\mathrm{J}_{\text {unbound }}$. 
TX-114 partitioning - The $\mathrm{D}$ and A phases were obtained from chromatography batches containing $6 \mathrm{mg}$ of total protein from the $\mathrm{J}_{\text {unbound }}$ fraction using TX-114 (Sigma), as described by Machado et al. (2007). J

was diluted in $1.7 \mathrm{~mL}$ of Tris-buffered saline $(10 \mathrm{mM}$ Tris- $\mathrm{HCl}, \mathrm{pH} 7.4,150 \mathrm{mM} \mathrm{NaCl}$ ) and $1 \% \mathrm{TX}-114$. This mixture was incubated at $0^{\circ} \mathrm{C}$ for $10 \mathrm{~min}$ and layered carefully on a sucrose mixture $(5.5 \mathrm{~mL}$ Tris-buffered saline plus $6 \%$ sucrose and $0.06 \% \mathrm{TX}-114)$. The mixture was incubated at $37^{\circ} \mathrm{C}$ for $10 \mathrm{~min}$, followed by centrifugation at $3,000 \mathrm{~g}$ and $25^{\circ} \mathrm{C}$ for $10 \mathrm{~min}$, after which the upper phase was collected, $1 \%$ TX-114 was added and the mixture was incubated at $0^{\circ} \mathrm{C}$ for $10 \mathrm{~min}$. This solution was again layered carefully on the sucrose mixture used previously, incubated at $37^{\circ} \mathrm{C}$ for $10 \mathrm{~min}$ and centrifuged at $3,000 \mathrm{~g}$ and $25^{\circ} \mathrm{C}$ for $10 \mathrm{~min}$. The supernatant was used to obtain the A phase and the pellet was used to obtain the D phase. The recovered supernatant was rinsed with $2 \%$ TX-114 in a separate tube without a sucrose cushion, incubated at $0^{\circ} \mathrm{C}$ for $10 \mathrm{~min}$ and then $37^{\circ} \mathrm{C}$ for $10 \mathrm{~min}$ and centrifuged at $3,000 \mathrm{~g}$ and $25^{\circ} \mathrm{C}$ for $10 \mathrm{~min}$. The supernatant of this solution consisted of the A phase. The fractionated components (the $\mathrm{DJ}_{\text {unbound }}$ and $\mathrm{AJ}_{\text {unbound }}$ fractions) were precipitated $(1: 2 ; \mathrm{v} / \mathrm{v})$ in cold acetone at $4^{\circ} \mathrm{C}$ overnight and centrifuged at $3,000 \mathrm{~g}$ and $4^{\circ} \mathrm{C}$ for $30 \mathrm{~min}$. The supernatants were discarded and the precipitates were resuspended in $1 \mathrm{~mL}$ Tris-buffered saline. The protein content of both $\mathrm{DJ}_{\text {unbound }}$ and $\mathrm{AJ}_{\text {unbound }}$ antigen preparations was determined according to Lowry et al. (1951).

SDS-PAGE - The T. solium metacestode fractions were diluted $(\mathrm{v} / \mathrm{v})$ in sample buffer and after boiling at $98^{\circ} \mathrm{C}$ for $3 \mathrm{~min}$, all antigen preparations and molecular weight markers (Sigma) were subjected to $12 \%$ SDSPAGE under non-reducing conditions, as described by Laemmli (1970), using an electrophoresis apparatus (Omniphor, England). The fractions and molecular weight markers were visualised by silver staining according to Friedman (1982) and the relative molecular masses were estimated using a logarithmic plot of the migration of a set of molecular weight standards, which was included in each gel.

ELISA - ELISAs for all extracts were carried out according to Machado et al. (2007). Briefly, polystyrene microplates (Interlab, Brazil) were coated with each extract at concentrations of $10 \mu \mathrm{g} / \mathrm{mL}$ in $0.06 \mathrm{M}$ carbonate bicarbonate buffer, $\mathrm{pH}$ 9.6. These preparations were incubated overnight at $4^{\circ} \mathrm{C}$ in a final volume of $50 \mu \mathrm{L} /$ well. The microplates were washed three times $(5 \mathrm{~min}$ each wash) with PBS containing $0.05 \%$ PBS-Tween 20 (PBS-T). The serum samples were diluted 1:200 in PBS-T, added to the microplates and incubated for 45 $\min$ at $37^{\circ} \mathrm{C}$. After washing as described above, peroxidase-conjugated goat anti-human $\operatorname{IgG}$ (Fc specific, Sigma) was added at a dilution of 1:1000 in PBS-T for all antigens and incubated for $45 \mathrm{~min}$ at $37^{\circ} \mathrm{C}$. The assay was read after washing by adding the enzymatic substrate solution, which consisted of $\mathrm{H}_{2} \mathrm{O}_{2}$ and ortho-phenylenediamine in $0.1 \mathrm{M}$ citrate phosphate buffer, $\mathrm{pH}$ 5.5, for $15 \mathrm{~min}$ followed by $25 \mu \mathrm{L} /$ well of $\mathrm{H}_{2} \mathrm{SO}_{4}(2 \mathrm{~N})$ to stop the reaction. Optical densities (OD) were determined at
$492 \mathrm{~nm}$ in an ELISA plate reader (Tp Reader, Thermoplate, China). The cut-off values were established using the mean OD of three non-reactive serum samples plus two standard deviations. The reactivity index (RI) was calculated according to Pardini et al. (2002). The data were subjected to "two-graph receiver operating characteristic" analysis, which is a plot of the test sensitivity and specificity against the threshold (cut-off) value, assuming the latter is an independent variable. A cut-off value was calculated for each antigen as the intersection point of the two graphs (Greiner et al. 1995). All serum samples with RI values $>1.1$ were considered positive.

Immunoblot - Immunoblot assays were performed on all ELISA-reactive serum samples according to Machado et al. (2007). Antigen preparations were subjected to electrophoresis and transferred to nitrocellulose membranes $(0.45 \mu \mathrm{m}$, Sigma), as previously described by Towbin et al. (1979), using a transfer apparatus (Omniphor, England). The nitrocellulose strips were blocked with $5 \%$ non-fat milk in PBS-T (PBS-TM) for $2 \mathrm{~h}$ at room temperature (RT) and incubated with serum samples diluted 1:50 in $1 \%$ PBS-TM overnight at $4^{\circ} \mathrm{C}$. After six washes with $1 \%$ PBS-TM, the strips were incubated for $2 \mathrm{~h}$ at RT with peroxidase-labelled goat IgG anti-human IgG (whole molecule, Sigma) diluted 1:1500 in 1\% PBS-TM. The strips were washed in PBS and developed for $3 \mathrm{~min}$ in the substrate solution, which contained hydrogen peroxide and 3,3-diaminobenzidine tetrahydrochloride (Sigma) in PBS. The reaction was stopped by washing with distilled water and positive reactions were identified by the appearance of clearly defined bands. The relative molecular masses of the recognised bands were determined by comparison with molecular markers (Sigma) and the serum samples were considered positive when immunodominant components with a molecular weight of 50-70 kDa were recognised, according to Machado et al. (2007).

Statistical analysis - The geometric means of titres (GMT) of the RI for the ELISA data were calculated and compared for each group of serum samples and antigenic extracts using Student's $t$ test. To determine if the proportion of positive samples within each group was significantly different from those of the other groups, the chi-square test was applied. Differences were considered significant when $p<0.05$. Sensitivity, specificity and diagnostic efficiency (DE) were calculated according to Barbieri et al. (1998) and the Youden Index (YI) was determined as described by Youden (1950).

\section{RESULTS}

Analysis of the extracts in SDS-PAGE - Fig. 1 shows the affinity chromatography and electrophoretic profile of the saline extract and fractions. The $J_{\text {unbound }}$ fraction showed various bands by SDS-PAGE $(87,78,70-50,45$, 37 and $24 \mathrm{kDa}$ ) and the jacalin-bound fraction showed five bands $(94,80,32,28$ and $14 \mathrm{kDa})$. The protein yield from this process was $75.4 \%$ for the unbound fraction and $25.5 \%$ for the bound fraction. In the ELISA, the $\mathrm{J}_{\text {unbound }}$ fraction was found to be more specific than the jacalin-bound fraction in terms of detection of IgG towards $T$. solium. Thus, only $\mathrm{J}_{\text {unbound }}$ was subjected to TX- 
114 partitioning. The protein yield from this process was $28.5 \%$ for the DJ phase and $55.5 \%$ for the AJ phase.

Fig. 2 shows the electrophoretic profile of the different antigenic fractions extracted from $T$. solium metacestodes with TX-114. The samples were separated by SDS-PAGE and stained with silver. Bands of 70-50, 48, 40 and $14 \mathrm{kDa}$ were visualised in the $\mathrm{DJ}_{\text {unbound }}$ fraction and bands of $85,66,50,45$ and $24 \mathrm{kDa}$ were detected in the $\mathrm{AJ}_{\text {unbound }}$ fraction.

Sensitivity and specificity of ELISA and immunoblot for the detection of T. solium IgG antibodies - As demonstrated in Fig. 3, all samples were tested by ELISA using the three antigen preparations. Thirty-four $(85 \%)$ of the serum samples from Group 1 were positive for $T$. solium IgG antibodies in the $\mathrm{J}_{\text {unbound }}$ fraction, $37(92.5 \%)$ in the $\mathrm{DJ}_{\text {unbound }}$ fraction and $33(82.5 \%)$ in the $\mathrm{AJ}_{\text {unbound }}$ fraction.

In Group 2, 13 (21\%) of the serum samples were positive for T. solium $\mathrm{IgG}$ antibodies in the $\mathrm{J}_{\text {unbound }}$ fraction, 14 $(22.6 \%)$ in the $\mathrm{AJ}_{\text {unbound }}$ fraction and only six $(9.7 \%)$ in the $\mathrm{DJ}_{\text {unbound }}$ fraction. As shown in Table, cross-reactivity by ELISA for the serum samples in Group 2 was predominantly due to E. granulosus infection. All serum samples from Group 3 were negative for T. solium IgG antibodies in the $\mathrm{DJ}_{\text {unbound }}$ fraction. One (3.3\%) sample from this group tested positive for T. solium $\operatorname{IgG}$ antibodies in the $\mathrm{J}_{\text {unbound }}$ fraction and two $(6.7 \%)$ samples were positive in the $\mathrm{AJ}_{\text {unbound }}$ fraction (Fig. 3). In Groups 2 and 3, the ELISA revealed $T$. solium specificities of $84.8 \%$ for $\mathrm{J}$ $93.5 \%$ for DJ. sulted in YI values of $0.70,0.86$ and 0.65 for the $\mathrm{J}_{\text {unbound }}$, $\mathrm{DJ}_{\text {unbound }}$ and $\mathrm{AJ}_{\text {unbound }}$ fractions, respectively. The DE was determined to be $84.8 \%, 93.2 \%$ and $82.6 \%$ for the $\mathrm{J}$ $\mathrm{DJ}_{\text {unbound }}$ and $\mathrm{AJ}_{\text {unbound }}$ fractions, respectively.

The immunoblot assays revealed the presence of a wide range of protein components in $\mathrm{J}_{\text {unbound, }}$, which varied in size from 24-110 kDa, as shown in Fig. 4. When using the $\mathrm{DJ}_{\text {unbound }}$ fraction, we found that all serum samples from the patients in Group 1, who were diagnosed

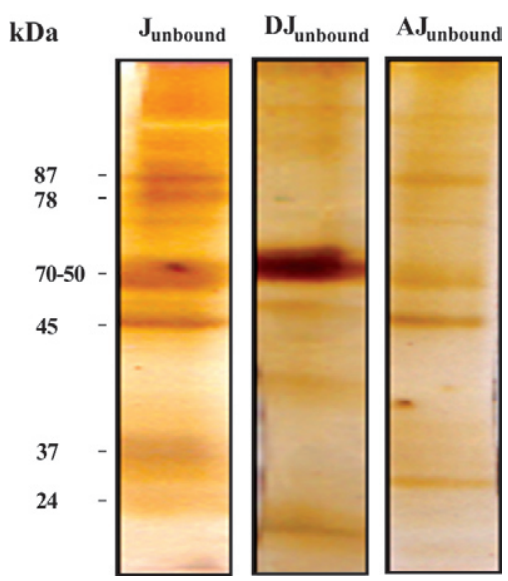

Fig. 2: electrophoretic profile of jacalin-unbound $\left(J_{\text {unbound }}\right)$ fraction from Taenia solium metacestodes and detergent ( $\left.\mathrm{DJ}_{\text {unbound }}\right)$ and aqueous $\left(\mathrm{AJ}_{\text {unbound }}\right)$ phases from this fraction obtained by partitioning with Triton X-114 in sodium dodecyl sulfate polyacrylamide gel electrophoresis at $12 \%$ by silver staining. Molecular mass standards are given on the left in $\mathrm{kDa}$.

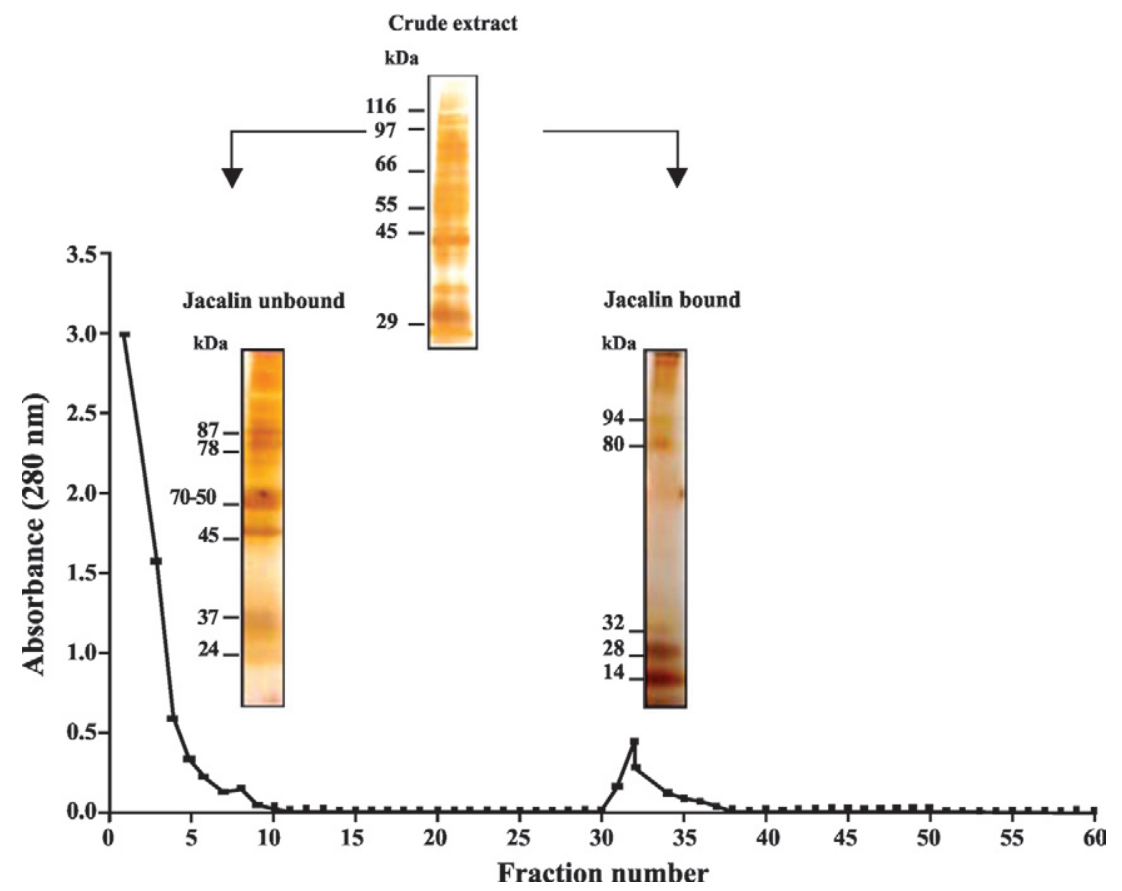

Fig. 1: affinity chromatography of saline extract of Taenia solium metacestodes in column of jacalin and electrophoretic profile of saline extract, jacalin-unbound and jacalin-bound in sodium dodecyl sulfate polyacrylamide gel electrophoresis at $12 \%$ by silver staining. Molecular mass standards for saline extract and molecular mass are given on the left in $\mathrm{kDa}$. 
with definitive NCC, reacted strongly with the 50-70 $\mathrm{kDa}$ component (Fig. 4A), whereas no reactivity was found for this component in the samples from Groups 2 and 3 (Fig. 4B, C). Poor reactivity was observed for all groups of serum samples using the $\mathrm{AJ}_{\text {unbound }}$ fraction, with the predominant reactivity occurring against the high molecular weight components (Fig. 4A-C).

\section{DISCUSSION}

In the present paper, we describe the partitioning of saline extracts from T. solium metacestodes using jacalin affinity chromatography followed by fractionation with TX-114, demonstrating for the first time that the D phase from the $\mathrm{J}_{\text {unbound }}$ fraction contains the antigenic components necessary for an accurate diagnosis of human NCC.

Purification by affinity chromatography resulted in $\mathrm{J}_{\text {unbound }}$ and jacalin-bound fractions. Distinct electrophoretic profiles were observed for these two fractions; the $\mathrm{J}_{\text {unbound }}$ fraction contained more components than the jacalin-bound fraction. The antigenic components with affinity to jacalin showed a fewer number of bands when compared to $T$. solium saline extract, but these components failed to provide sufficient information for the diagnosis of NCC.

The procedure that we describe in this paper, which utilises TX-114 phase partitioning, is fast, reproducible and relatively easy to perform because it does not require any specialised apparatuses or ultracentrifugation steps. The major advantages of using antigenic fractions purified with TX-114 are low production cost, simplic- ity and ease of extraction, high efficiency, speed and low toxicity from the lack of organic solvents required (Machado et al. 2007).

\section{TABLE}

Reactivity of serum samples from patients with other parasitoses (Group 2, $n=62$ ) for detection of $\operatorname{IgG}$ to jacalin-unbound ( $\left.\mathrm{J}_{\text {unbound }}\right)$ fraction, detergent ( $\mathrm{DJ}$ unbound and aqueous $\left(\mathrm{AJ}_{\text {unbound }}\right)$ phases from $\mathrm{J}_{\text {ubbound }}$ fraction from Taenia solium metacestodes by ELISA

\begin{tabular}{lccc}
\hline & \multicolumn{3}{c}{ Antibody detection } \\
\cline { 2 - 4 } & \multicolumn{3}{c}{ Positive samples } \\
& \multicolumn{3}{c}{$\mathrm{n}(\%)$} \\
\cline { 2 - 4 } & $\mathrm{J}_{\text {unbound }}$ & $\mathrm{DJ}_{\text {unbound }}$ & $\mathrm{AJ}_{\text {unbound }}$ \\
Infection (n) & $1(16.7)$ & $0(0)$ & $0(0)$ \\
Ascaris lumbricoides (6) & $10(100)$ & $6(60)$ & $9(90)$ \\
Echinococcus granulosus (10) & $0(0)$ & $0(0)$ & $1(16.7)$ \\
Enterobius vermicularis (6) & $0(0)$ & $0(0)$ & $1(25)$ \\
Giardia lamblia (4) & $0(0)$ & $0(0)$ & $0(0)$ \\
Hookworm (6) & $0(0)$ & $0(0)$ & $2(50)$ \\
Hymenolepis nana (4) & $0(0)$ & $0(0)$ & $0(0)$ \\
Schistosoma mansoni (10) & $1(25)$ & $0(0)$ & $1(25)$ \\
Strongyloides stercoralis (4) & $1(10)$ & $0(0)$ & $0(0)$ \\
Taenia sp (10) & $0(0)$ & $0(0)$ & $0(0)$ \\
Trichuris trichiura (2) & &
\end{tabular}

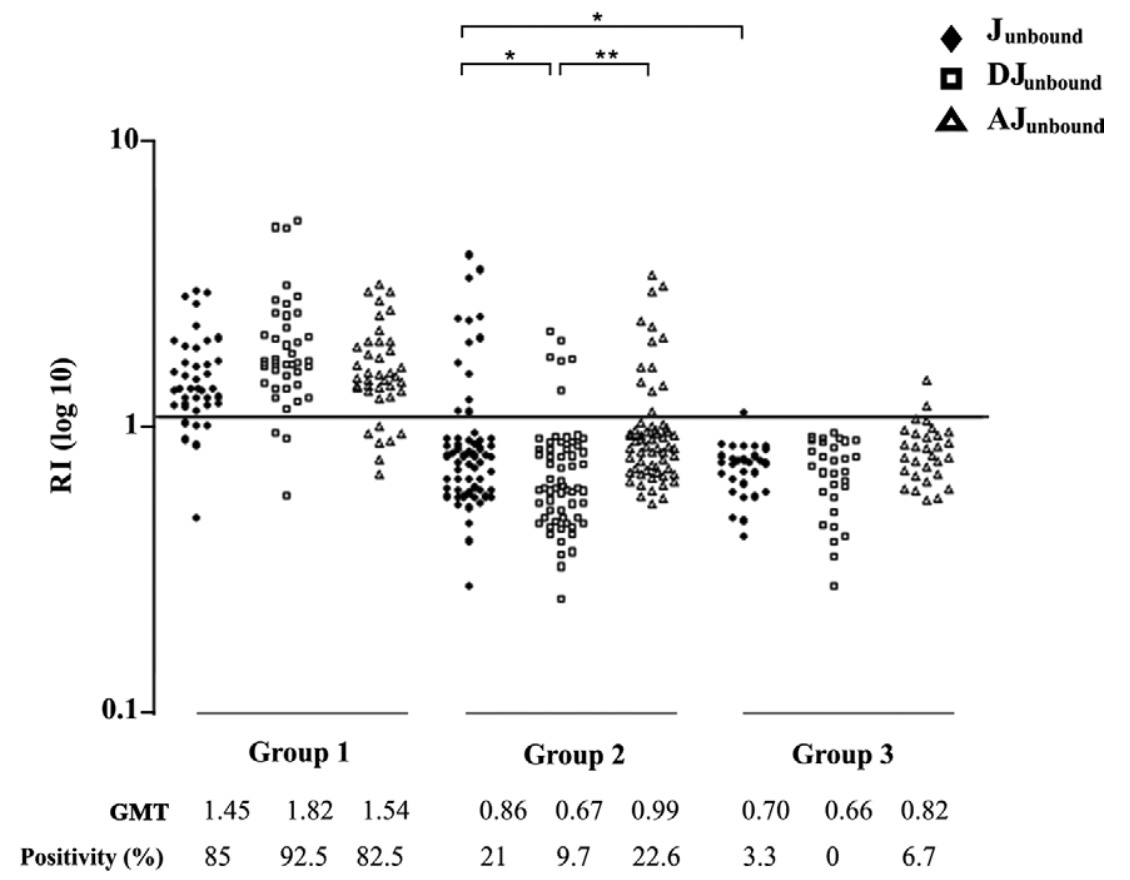

Fig. 3: detection of IgG antibodies anti-Taenia solium metacestodes in serum samples from patients with a definitive diagnosis of neurocysticercosis (Group 1; $\mathrm{n}=40$ ), other parasitoses (Group 2; $\mathrm{n}=62$ ) and apparently healthy individuals (Group 3; $\mathrm{n}=30$ ) by ELISA using the jacalinunbound $\left(\mathrm{J}_{\text {unbound }}\right)$ fraction from T. solium metacestodes and detergent $\left(\mathrm{DJ}_{\text {unbound }}\right)$ and aqueous $\left(\mathrm{AJ}_{\text {unbound }}\right)$ phases of this fraction. The horizontal line indicates the cut-off [reactivity index $(\mathrm{RI})>1.1$ ]. GMT: geometric means of titres; *: $\mathrm{p}<0.05 ; * *$ : $\mathrm{p}<0.005$. 
In the present study, jacalin affinity chromatography followed by TX-114 treatment proved to be adequate for fractionating the saline extract. When tested by ELISA, although $60 \%$ of the serum samples from patients with E. granulosus infections were cross-reactive, the $\mathrm{DJ}_{\text {un- }}$

fraction was more positive for T. solium IgG antibodies than the other fractions, as none of the samples from healthy individuals or those presenting other parasitoses exhibited any reactivity, indicating a significant YI and DE. The group of patients with other parasitoses is expected to represent the general population, particularly in developing countries where parasitic diseases are highly prevalent. Therefore, cross-reactivity may occur in screening tests such as ELISA, whose results would require further confirmation using an alternative immunoassay with more specific antigens.

When serum samples from patients with both NCC or E. granulosus infection were tested by the immunoblot assays performed on the $\mathrm{J}_{\text {unbound }}$ fraction, it was observed a reactivity pattern that included many bands, which contributes to the complication of a differential diagnosis. In contrast, when using the $\mathrm{DJ}_{\text {unbound }}$ fraction in the immunoblot assay, six serum samples from E. granulosusinfected patients did not show any reactivity for $T$. solium
IgGs and were therefore considered negative, although all of them had been considered positive by ELISA when using this antigenic fraction. Thus, the use of the $\mathrm{DJ}_{\text {unbound }}$ fraction in immunoblot assays allows for a differential diagnosis between NCC and E. granulosus infection. This is a particularly significant finding because these two types of infections constitute a major cause of laboratory diagnostic ambiguity due to their cross-reactivity in various immunoassays. The $50-70 \mathrm{kDa}$ band is an important component in the diagnosis of NCC. In fact, the proteins with a molecular weight of $50 \mathrm{kDa}$ have been reported previously, indicating high levels of sensitivity and specificity to the diagnosis of NCC (Hancock et al. 2004, Barcelos et al. 2007, Machado et al. 2007).

The $\mathrm{DJ}_{\text {unbound }}$ fraction showed $92.5 \%$ sensitivity and $93.5 \%$ specificity for T. solium IgGs, which are similar to the values reported by other authors who have used different techniques of antigen purification for NCC diagnosis (Pardini et al. 2002, Hancock et al. 2006, Machado et al. 2007). Moreover, the $\mathrm{DJ}_{\text {unbound }}$ fraction provided superior results to those obtained by other authors using whole extracts, where the sensitivity ranged from $70-85 \%$ and the specificity ranged from $80-88 \%$ (Bueno et al. 2000, Shiguekawa et al. 2000) for the detection of

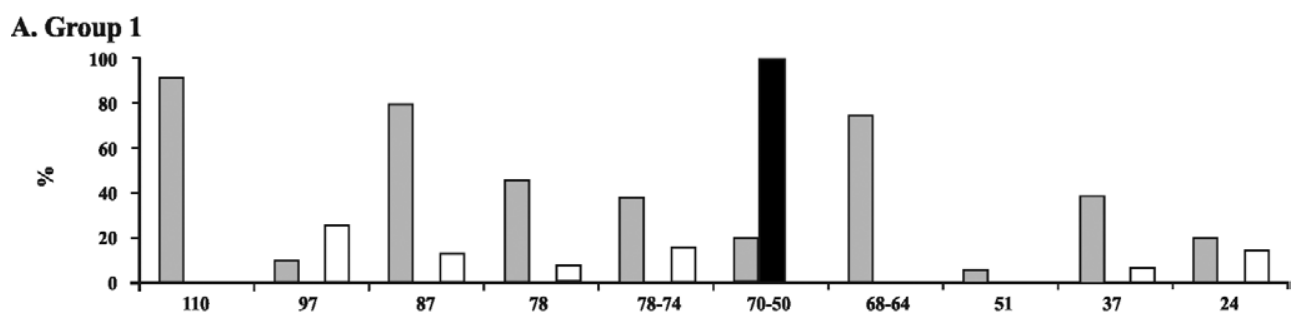

B. Group 2

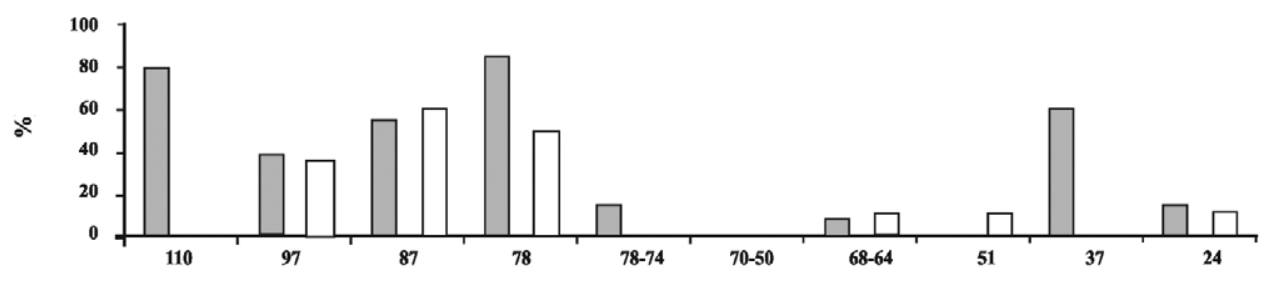

C. Group 3

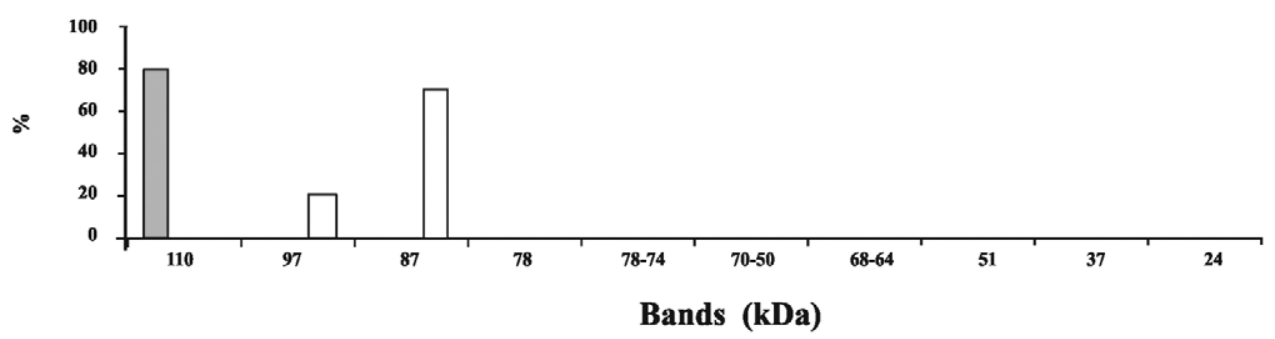

$\square \mathbf{J}_{\text {unbound }}$
$\square \mathbf{D J}_{\text {unbound }}$
$\square \mathbf{A J}_{\text {unbound }}$

Fig. 4: percentage of reactivity frequency of serum samples ELISA reactive by immunoblot using jacalin-unbound ( $\mathrm{J}_{\text {unbound }}$ ) fraction from Taenia solium metacestodes and detergent $\left(\mathrm{DJ}_{\text {unbound }}\right)$ and aqueous $\left(\mathrm{AJ}_{\text {unbound }}\right)$ of this fraction. A (Group 1): patients with definitive diagnosis of neurocysticercosis; B (Group 2): patients with other parasitoses; C (Group 3): apparently healthy individuals. 
anti-T. solium metacestodes IgG by ELISA. Although saline extracts have been widely used for primary screening in endemic areas, the identification and purification of highly specific glycoproteins associated with $T$. solium metacestodes have been the main focus for the serodiagnosis of NCC (Ito 2002, Lee et al. 2011). It is important to make clear that the other investigators used panels of lectins in order to identify NCC antigens and the jacalin was included in those panels (Trindade et al. 2006, Zhang et al. 2006). However, there is no previous study proposing the strategy to perform a jacalin affinity chromatography method and subsequent fractionating process by carry out a TX-114 partitioning from jacalin unbound fraction in the diagnosis of human NCC. Our results, however, demonstrate that a better diagnosis is obtained using the $\mathrm{DJ}_{\text {unbound }}$ fraction, indicating that if glycosylated antigens are present, they should not present galactosyl residues, as the isolated components do not show affinity for jacalin, a glycoprotein known to bind only to O-glycosidically linked oligosaccharides and to prefer to bind to galactosyl (b-1,3) N-acetylgalactosamine.

Considering the immunoassay design described here and the advantages of using the $\mathrm{DJ}_{\text {unbound }}$ fraction as the diagnostic component in immunoblot assays, it can be assumed that this assay can also be efficiently used to identify individuals with residual antibodies, as the presence of calcified metacestodes is common in endemic areas. The $50-70 \mathrm{kDa}$ band visualised in the $\mathrm{D}$ phase by immunoblot may improve the specificity of the tests, especially in serum samples, avoiding cross-reactivity and nonspecific reactions (Ishida et al. 2003).

In conclusion, the results obtained by immunoblot confirm that unbound antigenic fractions purified from T. solium metacestodes by jacalin chromatography followed by TX-114 treatment, especially $\mathrm{DJ}_{\text {unbound, }}$, are important putative sources of specific antigens for diagnostic kits and have a high value for the differential immunodiagnosis of NCC.

\section{ACKNOWLEDGEMENTS}

To Paula Cristina Brígido (ICBIM-UFU), for helping with figures preparations.

\section{REFERENCES}

Afonso SM, Vaz Y, Neves L, Pondja A, Dias G, Willingham AL, Vilhena M, Duarte PC, Jost CC, Noormahomed EV 2011. Human and porcine Taenia solium infections in Mozambique: identifying research priorities. Anim Health Res Rev 12: 123-129.

Agapejev S 2011. Neurocysticercosis: the enigmatic disease. Cent Nerv Syst Agents Med Chem 11: 261-284.

Baermann G 1917. Eine einfache nethods zur Auffindung von Ankylostomum (Nematoden) larven in endproben, Mededeel mit $\mathrm{h}$. Geneesk, La. Weltevreden Feestbundel, Batavia, p. 41-47.

Barbieri M, Fernández V, González G, Luaces VM, Nieto A 1998. Diagnostic evaluation of a synthetic peptide derived from a novel antigen $B$ subunit as related to other available peptides and native antigens used for serology of cystic hydatidosis. Parasite Immunol 20: 51-61.

Barcelos ISC, de Moura LP, da Costa VP, Ferreira MS, Costa-Cruz JM 2007. Taenia solium metacestode immunodominant peptides recognized by IgG antibodies in cerebrospinal fluid and serum paired samples from patients with active and inactive neurocysticercosis. Mem Inst Oswaldo Cruz 102: 713-717.

Barcelos ISC, Souza MA, Pena JDO, Machado GA, de Moura LGM, Costa-Cruz JM 2012. Genetic polymorphism in Taenia solium metacestodes from different Brazilian geographic areas. $\mathrm{Mem}$ Inst Oswaldo Cruz 107: 24-30.

Bordier C 1981. Phase separation of integral membrane proteins in Triton X-114 solution. J Biol Chem 256: 1604-1607.

Bueno EC, Vaz AJ, Machado LR, Livramento JA, Mielle SR 2000. Specific Taenia crassiceps and Taenia solium antigenic peptides for neurocysticercosis immunodiagnosis using serum samples. J Clin Microbiol 38: 146-151.

Carabin H, Ndimubanzi PC, Budke CM, Nguyen H, Qian Y, Cowan LD, Stoner JA, Rainwater E, Dickey M 2011. Clinical manifestations associated with neurocysticercosis: a systematic review. PLoS Negl Trop Dis 5: e1152.

Costa JM, Ferreira AW, Makino MM, Camargo ME 1982. Spinal fluid immunoenzymatic assay (ELISA) for neurocysticercosis. Rev Inst Med Trop Sao Paulo 24: 337-341.

Deckers N, Dorny P 2010. Immunodiagnosis of Taenia solium taeniosis/cysticercosis. Trends Parasitol 26: 137-144.

Del Brutto OH 2012. Neurocysticercosis: a review. Scientific WorldJournal doi: 10.1100/2012/159821.

Ferrer E, Sánchez J, Milano A, Alvarez S, La Rosa R, Lares M, González LM, Cortéz MM, Dávila I, Harrison LJ, Parkhouse RM, Gárate T 2012. Diagnostic epitope variability within Taenia solium $8 \mathrm{kDa}$ antigen family: implications for cysticercosis immunodetection. Exp Parasitol 130: 78-85.

Friedman RD 1982. Comparison of four different silver-staining techniques for salivary protein detection in alkaline poly-acrilamyde gels. Anal Biochem 126: 346-349.

Garcia HH, Gonzalez AE, Rodriguez S, Gonzalvez G, Llanos-Zavalaga F, Tsang VC, Gilman RH, Grupo de Trabajo en Cisticercosis en Perú 2010. Epidemiology and control of cysticercosis in Peru. Rev Peru Med Exp Salud Publica 27: 592-597.

Gonçalves FA, Machado GA, Oliveira HB, Rezende MT, Mineo JR, Costa-Cruz JM 2010. Hydrophobic fraction of Taenia saginata metacestodes, rather than hydrophilic fraction, contains immunodominant markers for diagnosing human neurocysticercosis. Rev Soc Bras Med Trop 43: 254-259.

Greiner M, Sohr D, Göbel P 1995. A modified ROC analysis for the selection of cut-off values and the definition of intermediate results of serodiagnostic tests. J Immunol Methods 185: 123-132.

Hancock K, Pattabhi S, Greene RM, Yushak ML, Williams F, Khan A, Priest JW, Levine MZ, Tsang VC 2004. Characterization and cloning of GP50, a Taenia solium antigen diagnostic for cysticercosis. Mol Biochem Parasitol 133: 115-124.

Hancock K, Pattabhi S, Whitfield FW 2006. Characterization and cloning of T24, a Taenia solium antigen diagnostic for cysticercosis. Mol Biochem Parasitol 147: 109-117.

Hermanson GT, Mattson GR, Krohn RI 1995. Preparation and use of immunoglobulin-binding affinity supports on emphaze beads. J Chromatogr A 691: 113-122.

Ishida MMI, Rubinsky-Elefant G, Ferreira AW, Hoshino-Shimizu S, Vaz AJ 2003. Helminth antigens (Taenia solium, Taenia crassiceps, Toxocara canis, Schistosoma mansoni and Echinococcus granulosus) and cross-reactivities in human infections and immunized animals. Acta Trop 89: 73-84.

Ito A 2002. Serologic and molecular diagnosis of zoonotic larval cestode infections. Parasitol Int 51: 221-235. 
Jeyaprakash AA, Jayashree G, Mahanta SK, Swaminathan CP, Sekar K, Surolia A, Vijayan M 2005. Structural basis for the energetics of jacalin-sugar interactions: promiscuity versus specificity. J Mol Biol 347: 181-188.

Kabir S 1998. Jacalin: a jackfruit Artocarpus heterophyllus/seedderived lectin of versatile applications in immunobiological research. J Immunol Methods 212: 193-211.

Laemmli UK 1970. Cleavage of structural proteins during the assembly of the head of bacteriophage T4. Nature 227: 680-685.

Lee YM, Handali S, Hancock K, Pattabhi S, Kovalenko VA, Levin A, Rodriguez S, Lin S, Scheel CM, Gonzalez AE, Gilman RH, Garcia HH, Tsang VC 2011. Serologic diagnosis of human Taenia solium cysticercosis by using recombinant and synthetic antigens in QuickELISATM. Am J Trop Med Hyg 84: 587-593.

Lowry VH, Rosebrouch NJ, Farr AL, Randal RJ 1951. Protein measurement with the folin phenol reagent. J Biol Chem 193: 265-275.

Lutz A 1919. Schistosomum mansoni and schistosomiasis observed in Brazil. Mem Inst Oswaldo Cruz 11: 109-140.

Machado GA, Santiago FM, Mineo JR, Costa-Cruz JM 2007. Assessment of antigenic fractions of varying hydrophobicity from Taenia solium metacestodes for the diagnosis of human neurocysticercosis. Trop Med Int Health 12: 1369-1376.

Michelet L, Fleury A, Sciutto E, Kendjo E, Fragoso G, Paris L, Bouteille B 2011. Human neurocysticercosis: comparison of different diagnostic tests using cerebrospinal fluid. J Clin Microbiol 49: 195-200.

Nash TE, Garcia HH 2011. Diagnosis and treatment of neurocysticercosis. Nat Rev Neurol 13: 584-594.

Pardini AX, Peralta RH, Vaz AJ, Machado LD, Peralta JM 2002. Use of Taenia crassiceps cysticercus antigen preparations for detection of antibodies in cerebrospinal fluid samples from patients with neurocysticercosis (Taenia solium). Clin Diagn Lab Immunol 9: 190-193.

Prasad KN, Verma A, Srivastava S, Gupta RK, Pandey CM, Paliwal VK 2011. An epidemiological study of asymptomatic neurocysticercosis in a pig farming community in northern India. Trans R Soc Trop Med Hyg 105: 531-536.

Ritchie LS 1948. An ether sedimentation technique for routine stool examinations. Bull U S Army Med Dep 8: 326-334.

Shiguekawa KYM, Mineo JR, Moura LP, Costa-Cruz JM 2000. ELI$\mathrm{SA}$ and western blotting tests in the detection of IgG antibodies to Taenia solium metacestodes in serum samples in human neurocysticercosis. Trop Med Int Health 5: 443-449.

Sotelo J, Guerrero V, Rubio F 1985. Neurocysticercosis: a new classification based on active and inactive forms. A study of 753 cases. Arch Intern Med 145: 442-445.

Towbin H, Staehelin T, Gordon J 1979. Electrophoretic transfer of proteins from polyacrylamide gels to nitrocellulose sheets: procedure and some applications. Proc Natl Acad Sci USA 76: 4350-4354.

Trindade MB, Lopes JLS, Soares-Costa A, Monteiro-Moreira AC, Moreira RA, Oliva MLV, Beltramini LM 2006. Structural characterization of novel chitin-binding lectins from the genus Artocarpus and their antifungal activity. Biochim Biophys Acta 1764: 146-152.

Wu AM, Wu JH, Lin LH, Lin SH, Liu JH 2003. Binding profile of $A r$ tocarpus integrifolia agglutinin (Jacalin). Life Sci 72: 2285-2302.

Yanagida T, Sako Y, Nakao M, Nakaya K, Ito A 2012. Taeniasis and cysticercosis due to Taenia solium in Japan. Parasit Vectors 5: 18.

Youden WJ 1950. Index for rating diagnosis tests. Cancer 3: 32-35.

Zhang JJ, Xu LX, ZhangY, Zhao MH 2006. Binding capacity of in vitro deglycosylated Ig A1 to human mesangial cells. Clin Immunol 119: 103-109. 